\title{
On the Optimization of Derivative-Based Receivers for Molecular Communications
}

\author{
Mustafa Can Gursoy \\ University of Southern California \\ Department of Electrical and Computer Engineering \\ Los Angeles, USA \\ mgursoy@usc.edu
}

\author{
Urbashi Mitra \\ University of Southern California \\ Department of Electrical and Computer Engineering \\ Los Angeles, USA \\ ubli@usc.edu
}

\begin{abstract}
Inter-symbol interference (ISI) due to random molecule propagation challenges achieving high data-rate diffusion-based molecular communication (DBMC) systems. Recently, pre-processing the received signal by higher order derivatives has shown to provide an aggressive right tail suppression of the channel impulse response resulting in efficient ISI mitigation, and enabling the achievement of an order of magnitude increase in data rate while preserving reliability. However, the gains of derivative-based DBMC receivers are heavily dependent on the proper selection of the derivative order. In order to further improve the efficacy of derivative-based DBMC receivers, detector design and derivative order optimization is addressed in this study. Herein, the well-known fixed sample, fixed threshold detector (FSTD) detector is generalized to work with the derivative operator. For the derivative operator-FSTD pair, a signal-to-interference-and-noise ratio-like (SINR) cost function is introduced to optimize the derivative order. Numerical results confirm the accuracy of the SINR in derivative order optimization, and the efficacy of the derivative-aided FSTD strategy in terms of error performance.
\end{abstract}

\section{CCS CONCEPTS}

- Mathematics of Computing $\rightarrow$ Information theory; Probability and Statistics.

\section{KEYWORDS}

diffusion-based molecular communications, higher order derivatives, receiver pre-processing, detection

\section{ACM Reference Format:}

Mustafa Can Gursoy and Urbashi Mitra. 2021. On the Optimization of Derivative-Based Receivers for Molecular Communications. In The Eight Annual ACM International Conference on Nanoscale Computing and Communication (NANOCOM '21), September 7-9, 2021, Virtual Event, Italy. ACM, New York, NY, USA, 6 pages. https://doi.org/10.1145/3477206.3477452

Permission to make digital or hard copies of all or part of this work for personal or classroom use is granted without fee provided that copies are not made or distributed for profit or commercial advantage and that copies bear this notice and the full citation on the first page. Copyrights for components of this work owned by others than ACM must be honored. Abstracting with credit is permitted. To copy otherwise, or republish, to post on servers or to redistribute to lists, requires prior specific permission and/or a fee. Request permissions from permissions@acm.org.

NANOCOM '21, September 7-9, 2021, Virtual Event, Italy

(c) 2021 Association for Computing Machinery.

ACM ISBN 978-1-4503-8710-1/21/09..\$15.00

https://doi.org/10.1145/3477206.3477452

\section{INTRODUCTION}

Diffusion-based molecular communication (DMBC) systems suffer from significant inter-symbol interference (ISI) that hinders achieving high data rate and low error rate communication [4]. Motivated by this, many transmitter and receiver side solutions have been considered to alleviate the effects of ISI. The transmitter side schemes mainly focus on modulation design [2, 7, 12], coding [17], and pre-equalization $[14,18]$, whereas the receiver side strategies include detector design $[3,15]$, equalization algorithms $[9,11]$, and synchronization approaches [19].

Recently, as a receiver side solution to the ISI mitigation problem, operating on the first order time derivative of the received signal (as opposed to the received signal itself) was proposed in $[8,20]$. This approach is generalized to an arbitrary derivative order $m$ in our prior work [5], where we had introduced a general framework for derivative-based DBMC received signal processing, characterized its main trade-off, and discussed several detector schemes to be paired with the derivative operator. This paper extends and improves on our previous study from a detector design perspective, and proposes a solution to the derivative order optimization problem. Overall, the main contributions of this study over the state-of-the-art and our previous work are as follows:

- In contrast to using the max-and-threshold detector (MaTD) employed in prior work $([5,20])$, we generalize the wellknown fixed sample, fixed threshold detector (FSTD), and propose its use in conjunction with the $m^{t h}$ order derivative operator.

- Recognizing that the derivative order $m$ is a parameter to be optimized, we provide a signal-to-interference and noise ratio-like (SINR) cost function to optimize the derivative order $m$.

- Our numerical results show that the FSTD provides reliable communication at high data rates, while still keeping the simplicity of a fixed threshold detector. Combining with the easy-to-implement derivative operation, the $m^{t h}$ order derivative \& FSTD pair provides high data rates with low error rates, with a complexity that is amenable to micro- to nano-scale implementation.

The rest of the paper is organized as follows: Section 2 describes the considered channel model and received signal statistics. Section 3 presents the characteristics and the main trade-off of a derivativebased DBMC receiver. Section 4 introduces the proposed derivative $\&$ detector pair. Section 5 derives and provides a cost function to be used when optimizing the derivative order, $m$. Section 6 presents the numerical results, and Section 7 concludes the paper. 


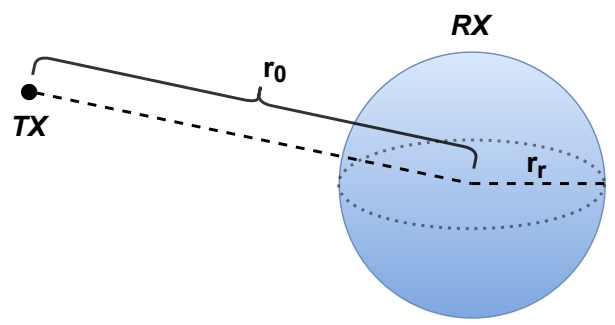

Figure 1: The considered communication environment.

\section{SYSTEM MODEL}

In this paper, we consider a molecular communication link between a point transmitter and a synchronized, spherical receiver in a 3-D, unbounded environment as presented in Figure 1, similar to the considerations of [22]. Throughout the paper, the distance between the point transmitter and the spherical receiver's center is denoted by $r_{0}$, the receiver's radius is denoted by $r_{r}$, and the diffusion coefficient of the carrier molecules is denoted by $D$.

For our topology of interest, the time arrival density function of the molecules (i.e., the channel impulse response, CIR) is computed by $[22]$ as

$$
f_{h i t}(t)=\frac{r_{r}}{r_{0}} \frac{1}{\sqrt{4 \pi D t}} \frac{r_{0}-r_{r}}{t} e^{-\frac{\left(r_{0}-r_{r}\right)^{2}}{4 D t}}, \quad t \in(0, \infty) .
$$

The time integral of (1) yields the fraction of arriving molecules up to time $t$, which is given by [22] as

$$
F_{h i t}(t)=\frac{r_{r}}{r_{0}} \operatorname{erfc}\left(\frac{r_{0}-r_{r}}{\sqrt{4 D t}}\right) .
$$

In this paper, we consider a time-slotted DBMC system. Such a system is characterized by the channel coefficient vector $\boldsymbol{h}$, where $h[n]$ defines the probability of a molecule arriving between the interval $\left((n-1) t_{s}, n t_{s}\right]$ time after release, where $t_{s}$ is the slot (sample) duration. The $n^{\text {th }}$ channel coefficient is found by

$$
h[n]=F_{h i t}\left(n t_{s}\right)-F_{h i t}\left((n-1) t_{s}\right), \quad n=1,2, \ldots, L N
$$

where $L$ denotes the channel memory in symbols, and $N$ denotes the number of samples per one symbol duration. We consider binary concentration shift keying (BCSK) signaling throughout the paper, where a bit-1 is transmitted by emitting $M$ molecules whilst a bit-0 is transmitted by no emission [13]. Note that this definition implies that for binary signaling, the bit (hence symbol) duration is $t_{b}=N t_{s}$.

We note that $\boldsymbol{h}$ describes the probabilities of a single molecule's arrivals. For a sequential symbol transmission scenario with multiple molecule emissions, the received signal $\boldsymbol{y}$ can be modeled by the linear time-invariant (LTI)-Poisson channel model [1], where

$$
y[n] \sim \mathcal{P}\left(\lambda_{s}+\sum_{k=1}^{L N} h[k] x[n-k+1]\right) .
$$

Here, $\mathcal{P}(\mu)$ denotes the Poisson distribution with rate parameter $\mu$, $\lambda_{s}$ is the rate of the external Poisson noise, and $x$ denotes the vector of emitted molecules. In this paper, we assume an idealized transmitter. Hence, the binary vector $s$ of transmitted bits deterministically defines $x$. Specifically, since we employ BCSK (with equiprobable bit transmissions) and consider emission at the beginning of the symbol duration,

$$
x[i]= \begin{cases}M, & \text { if } s[k]=1 \text { and } i=(k-1) N+1 \\ 0, & \text { otherwise. }\end{cases}
$$

Throughout this paper, we employ the Gaussian approximation of the Poisson arrivals [21]. Hence, by using vector notation, separating the deterministic and random components of $\boldsymbol{y}$, and considering a transmission block of length $S$, the received signal can be represented as

$$
\boldsymbol{y}=\left(\boldsymbol{H} \boldsymbol{x}+\lambda_{s} \boldsymbol{j}\right)+\boldsymbol{\eta},
$$

where $\boldsymbol{j}$ is a vector of ones, and $\boldsymbol{H}$ is the Toeplitz matrix describing the convolution operation in (4). Note that all vectors are of dimension $S N \times 1$ and $\boldsymbol{H}$ is an $S N \times S N$ matrix. Using the Gaussian approximation, the noise vector $\boldsymbol{\eta}$ (i.e., the random component of $\boldsymbol{y})$ is distributed as

$$
\begin{aligned}
\boldsymbol{\eta} & \sim \mathcal{N}(\mathbf{0}, \Sigma), \\
\Sigma & =\operatorname{diag}\{\boldsymbol{H} \boldsymbol{x}\}+\lambda_{s} \boldsymbol{I} .
\end{aligned}
$$

We note that the noise covariance matrix is affected by the emission sequence $x$, implying the well-known signal-dependent noise of DBMC systems $[6,10,11]$.

\section{THE DERIVATIVE PRE-PROCESSOR}

Herein, we discuss the key trade-off of applying an $m^{\text {th }}$ order derivative pre-processing on the received signal before the detection stage, by characterizing the operator's effects on received signal statistics. As the CIR function in (1) represents the expected signal of a one-shot transmission, we first investigate how the time derivatives of $f_{\text {hit }}(t)$ evolve with $m$, and introduce the main motivation of applying an $m^{t h}$ order derivative pre-processing.

As can also be observed from Figure 2, the $m^{\text {th }}$ order time derivative of the CIR (i.e., $\frac{\partial^{m} f_{h i t}}{\partial t^{m}}$ ) becomes less dispersive in time as $m$ increases. This corresponds to an effective pulse narrowing in the time slotted discrete time model presented in Section 2, which corresponds to a desirable ISI mitigation. Using the discrete time model and with matrix notation, the discrete-time forward derivative operator $D$ is defined as

$$
D=\left[\begin{array}{ccccc}
-1 & 1 & 0 & \cdots & 0 \\
0 & -1 & 1 & \cdots & 0 \\
\vdots & \vdots & \ddots & \ddots & \vdots \\
\vdots & \vdots & & -1 & 1 \\
0 & 0 & \ldots & 0 & -1
\end{array}\right] .
$$

Therefore, defining $\boldsymbol{y}_{(m)} \sim \mathcal{N}\left(\boldsymbol{\mu}_{(m)}, \Sigma_{(m)}\right)$ as the output of the $m^{t h}$ order derivative operator, we can write

$$
\begin{aligned}
\boldsymbol{y}_{(m)} & =\boldsymbol{D}^{m} \boldsymbol{y} \\
& =\boldsymbol{D}^{m}\left(\boldsymbol{H} \boldsymbol{x}+\lambda_{s} \boldsymbol{j}\right)+\boldsymbol{D}^{m} \boldsymbol{\eta} .
\end{aligned}
$$

Here, the application of the $D^{m}$ operator to the deterministic part of $\boldsymbol{y}$ introduces the aforementioned ISI mitigation. However, the expression $D^{m} \boldsymbol{\eta}$ introduces noise coloring and amplification to the received signal, since $\Sigma_{(m)}=D^{m} \Sigma\left(D^{\top}\right)^{m}$. Furthermore, the larger the derivative order $m$, the more severe the noise amplification 

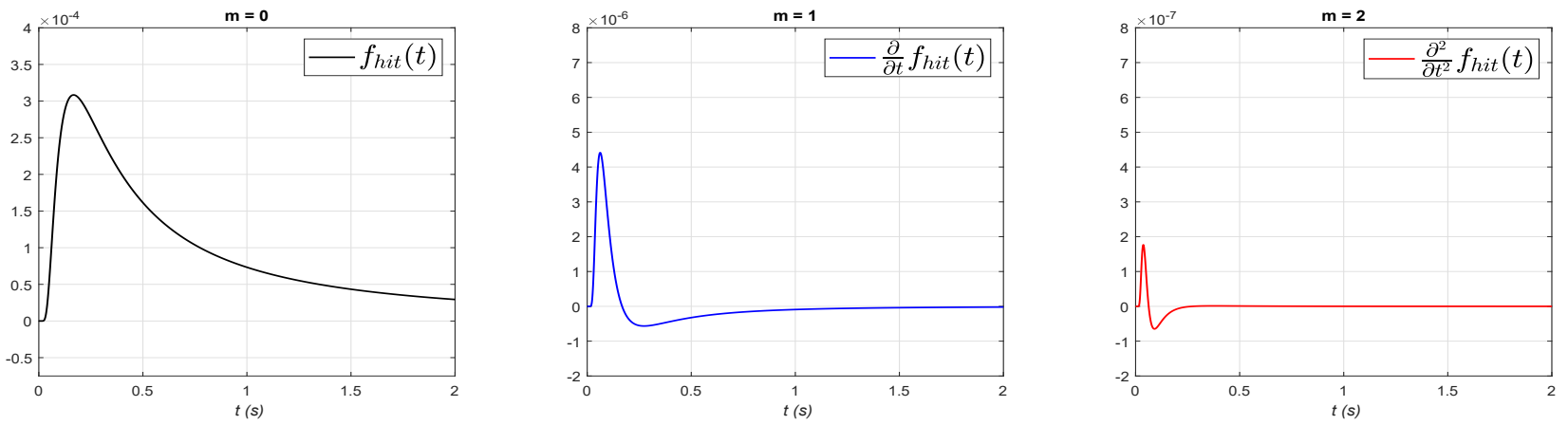

Figure 2: Evolution of $\frac{\partial^{m} f_{\text {hit }}}{\partial t^{m}}$ with the derivative order $m \cdot r_{0}=15 \mu \mathrm{m}, r_{r}=5 \mu \mathrm{m}, D=100 \frac{\mu \mathrm{m}^{2}}{\mathrm{~s}}$.

becomes. As also pointed out in [5], this introduces a fundamental trade-off between ISI mitigation and noise amplification for a derivative-based pre-processor, implying the existence of an optimal $m$ that minimizes the error probability. In Section 5 , we will address this optimization problem by devising an appropriate cost function for the system at hand.

\section{DETECTOR DESIGN}

As also mentioned in $[5,11]$, the ISI nature of the DBMC channel causes the maximum likelihood detector to be in the form a sequence detector. However, as a sequence detector has exponential complexity in channel memory $L$, we consider symbol-by-symbol, fixed threshold detectors that are amenable to nano-scale implementation. Conventionally, the fixed threshold detector for a derivativebased DBMC receiver is implemented by

- performing the derivative operation,

- discarding last $m$ samples to cancel non-causal ISI ${ }^{1}$,

- finding the maximum count among the remaining $(N-m)$ samples,

- comparing with a threshold $(\gamma)$,

respectively $([5,20])$. In other words, this max-and-threshold detector (MaTD) performs

$$
\hat{s}[i]=\max \left(y_{(m)}[(i-1) N+1], \cdots, y_{(m)}[i N-m]\right) \underset{0}{\stackrel{1}{\gtrless} \gamma,}
$$

which can be thought of as a generalization of the simple asynchronous detector proposed in [16]. We note that such a detector is inherently memoryless, which is beneficial in terms of simplicity. Furthermore, although we described $m^{\text {th }}$ order derivative operation is through a matrix multiplication for analysis purposes (i.e., $\left.\boldsymbol{y}_{(m)}=\boldsymbol{D}^{\boldsymbol{m}} \boldsymbol{y}\right)$, in reality it can be implemented by simple shift registers and adders. Combining the simple structures of both of these steps, the joint use of $\boldsymbol{D}^{m}$ and MaTD offers a very low complexity solution with good performance.

In this paper, we consider an alternative to MaTD to be coupled with the $D^{m}$ operator, where the maximum-finding operation is omitted, and the molecule count that is compared with a threshold is

\footnotetext{
${ }^{1}$ Note that the $m^{\text {th }}$ order forward derivative operation finds $y_{(m)}[i]$ as a function of $y[i], \ldots, y[i+m]$. For the last $m$ samples of a symbol, this operation introduces correlations with the first samples of the next symbol, hence inducing a non-causal ISI. Truncating the last $m$ samples avoids this issue.
}

taken from a fixed sample. We denote this sample by $\tilde{q}_{(m)}$. Recalling from Figure 2 that the peak time changes with $m$, we emphasize that $\tilde{q}_{(m)}$ is a function of $m$. We find $\tilde{q}_{(m)}$ by finding the sample that yields the largest expected arrival count resulting from the intended symbol's transmission, which can be expressed as

$$
\tilde{q}_{(m)}=\underset{q \in\{1, \ldots, N-m\}}{\arg \max }\left|\mu_{s,(m)}[q]\right|,
$$

where

$$
\boldsymbol{\mu}_{s,(m)}=D^{m} \boldsymbol{\mu}_{s}
$$

and the expected arrival count vector $\boldsymbol{\mu}_{s}$ can be found by

$$
\boldsymbol{\mu}_{s}=\left[\begin{array}{lll}
\frac{M}{2} h[1] & \ldots & \frac{M}{2} h[N] .
\end{array}\right]^{\top}
$$

Note that $\tilde{q}_{(m)} \in\{1, \ldots, N-m\}$, in order to avoid the non-causal ISI introduced by the forward derivative operation. Furthermore, Equation (11) picks the count that is largest in absolute sense, rather than performing an $\arg \max$ on $\boldsymbol{\mu}_{s,(m)}$ itself. This is due to the fact that after applying successive time differentiation, the resultant $\boldsymbol{\mu}_{s,(m)}$ can have negative elements that are actually larger in magnitude than the largest positive element. This implies a larger energy in said negative sample, therefore in a case where $\mu_{s,(m)}\left[\tilde{q}_{(m)}\right]<0$, we simply negate the received signal and operate accordingly. Therefore, the decision rule for FSTD becomes

$$
\hat{s}[i]= \begin{cases}y_{(m)}\left[(i-1) N+\tilde{q}_{(m)}\right] \gtrless_{0}^{1} \gamma, & \text { if } \mu_{s,(m)}\left[\tilde{q}_{(m)}\right] \geq 0 \\ -y_{(m)}\left[(i-1) N+\tilde{q}_{(m)}\right] \gtrless_{0}^{1} \gamma, & \text { if } \mu_{s,(m)}\left[\tilde{q}_{(m)}\right]<0,\end{cases}
$$

which is a generalization of the fixed sample, fixed threshold detector (FSTD) that is widely used in the DBMC literature to the $m^{\text {th }}$ order derivative.

\section{ON THE DERIVATIVE ORDER OPTIMIZATION}

In this section, by extending the signal-to-interference and noise ratio (SINR) employed by [10] to an arbitrary derivative order $m$ and tailoring it to our considered detector, we introduce a cost function that optimizes the derivative order for a $D^{m}$-FSTD pair. 
Overall, the proposed cost function has the following form:

$$
\operatorname{SINR}(m)=\frac{E\left\{\left(s[k] \mu_{s,(m)}\left[\tilde{q}_{(m)}\right]\right)^{2}\right\}}{\operatorname{Var}\left\{\eta_{(m)}^{(k)}\left[\tilde{q}_{(m)}\right]\right\}+\operatorname{Var}\left\{\mathcal{I}_{(m)}^{(k)}\left[\tilde{q}_{(m)}\right]\right\}} .
$$

Here,

$$
\begin{aligned}
E\left\{\left(s[k] \mu_{s,(m)}\left[\tilde{q}_{(m)}\right]\right)^{2}\right\} & =\frac{1}{2} 0+\frac{1}{2} E\left\{\left(\mu_{s,(m)}\left[\tilde{q}_{(m)}\right]\right)^{2}\right\} \\
& =\frac{1}{2}\left(\mu_{s,(m)}\left[\tilde{q}_{(m)}\right]\right)^{2},
\end{aligned}
$$

with $\boldsymbol{\mu}_{s,(m)}$ is as defined in Equation (12). Note that the expression in (16) corresponds to the second moment of the signal induced by the intended symbol. Since the $D^{m}$-FSTD pair considers the $\tilde{q}_{(m)}^{t h}$ sample, the calculation is performed on said sample.

In the denominator of (15), the first expression corresponds to the signal-dependent noise variance due to the intended symbol $s[k]$. This quantity corresponds to the $\tilde{q}_{(m)}^{t h}$ diagonal entry of the covariance matrix that is induced by the intended symbol, which can be expressed as

$$
\begin{aligned}
\operatorname{Var}\left\{\eta_{(m)}^{(k)}\left[\tilde{q}_{(m)}\right]\right\} & =\bar{\Sigma}_{s,(m)}\left[\tilde{q}_{(m)}, \tilde{q}_{(m)}\right] \\
\bar{\Sigma}_{s,(m)} & =\frac{1}{2}\left\{\boldsymbol{D}^{m} \operatorname{diag}\left(\boldsymbol{\mu}_{s,(m)}\right)\left(\boldsymbol{D}^{\top}\right)^{m}\right\} .
\end{aligned}
$$

Lastly, the second term in the denominator is the variance of the received signal at the $\tilde{q}_{(m)}^{t h}$ sample that is due to ISI. This value is the $\tilde{q}_{(m)}^{t h}$ diagonal entry of the covariance matrix induced by ISI, that is

$$
\operatorname{Var}\left\{\mathcal{I}_{(m)}^{(k)}\left[\tilde{q}_{(m)}\right]\right\}=\bar{\Sigma}_{I,(m)}\left[\tilde{q}_{(m)}, \tilde{q}_{(m)}\right]
$$

Considering the channel has a memory of $L$, the entries of this covariance matrix $\bar{\Sigma}$ can be calculated as follows;

$$
\begin{aligned}
& \bar{\Sigma}_{I,(m)}[i, j]=\operatorname{Cov}\left(y_{(m)}^{(k)}[i], y_{(m)}^{(k)}[j]\right) \\
& =E\left[\left(y_{(m)}^{(k)}[i] \quad y_{(m)}^{(k)}[j]\right]-E\left[y_{(m)}^{(k)}[i]\right] \quad E\left[y_{(m)}^{(k)}[j]\right]\right. \\
& =E_{\boldsymbol{s}_{I S I}}\left[E\left[\left(y_{(m)}^{(k)}[i] y_{(m)}^{(k)}[j] \mid \boldsymbol{s}_{I S I}\right]\right]\right. \\
& -E_{\boldsymbol{s}_{I S I}}\left[E\left[y_{(m)}^{(k)}[i] \mid \boldsymbol{s}_{\mathrm{ISI}}\right]\right] E_{\boldsymbol{s}_{I S I}}\left[E\left[y_{(m)}^{(k)}[j] \mid \boldsymbol{s}_{\mathrm{ISI}}\right]\right] \\
& =\frac{1}{2^{L-1}} \sum_{\forall s_{I S I}} \Sigma_{I,(m)}[i, j]+\mu_{I,(m)}[i] \mu_{I,(m)}[j] \\
& -\left(\frac{1}{2^{L-1}} \sum_{\forall s_{I S I}} \mu_{I,(m)}[i]\right)\left(\frac{1}{2^{L-1}} \sum_{\forall s_{I S I}} \mu_{I,(m)}[j]\right) .
\end{aligned}
$$

where $\boldsymbol{\mu}_{\boldsymbol{I}}$ is the induced mean vector conditioned on an ISI symbol sequence $s_{I S I}$, and

$$
\begin{aligned}
& \boldsymbol{\mu}_{I,(m)}=\boldsymbol{D}^{m} \boldsymbol{\mu}_{\boldsymbol{I}} \\
& \Sigma_{I,(m)}=\boldsymbol{D}^{m} \operatorname{diag}\left(\boldsymbol{\mu}_{\boldsymbol{I}}\right)\left(\boldsymbol{D}^{\top}\right)^{m} .
\end{aligned}
$$

Note that due to the exponential complexity in $L$ when computing (19), SINR can be evaluated using a smaller memory window $L^{\prime}<L$. As will the numerical results present in the sequel, this consideration still yields an accurate cost function in terms of reflecting the comparative trends in BER with respect to $m$. Therefore, denoting the memory-limited version of SINR as $\operatorname{SINR}_{L^{\prime}}$, the derivative order $m$ can be picked according to the following rule:

$$
m^{*}=\underset{m}{\arg \max } \operatorname{SINR}_{L^{\prime}}(m) .
$$

\section{NUMERICAL RESULTS}

Herein, we present numerical results to demonstrate the accuracy of the cost function presented in Section 5, and comparative bit error ratio (BER) results. Throughout the section, we normalize the power of the external noise rate $\lambda_{s}$ with respect to the transmission power of the transmitter by defining SNR as follows:

$$
\mathrm{SNR}=\frac{M}{2 N \lambda_{s}} .
$$

Since we assume equiprobable bit transmissions, the average number of molecules emitted per bit is equal to $\frac{M}{2}$, whereas $N \lambda_{s}$ corresponds to the external noise rate per one bit duration (recall $t_{b}=N t_{s}$ ). In addition, we normalize the bit duration (hence the data rate) in terms of the channel peak time $t_{\text {peak }}=\frac{d^{2}}{6 D}$, (see [22, Equation 26]), by considering a unitless parameter defined as

$$
S_{r}=\frac{t_{b}}{t_{\text {peak }}}
$$

We note that due to its definition, a smaller $S_{r}$ corresponds to a higher data rate.

\subsection{Accuracy of the Proposed SINR Metric}

Firstly, Figures $3 \mathrm{a}$ and $3 \mathrm{~b}$ are presented to demonstrate the accuracy of the SINR expression derived in Section 5. Note that as the computational complexity of evaluating (19) is exponential in channel memory, SINR is evaluated considering a significantly shorter memory of $L^{\prime}=10$ (i.e., $\operatorname{SINR}_{L^{\prime}}$ ), in contrast to the true channel memories of $L=100$ for Figure 3c and $L=200$ for Figure 3d.

Comparing Figures $3 \mathrm{a}$ and $3 \mathrm{~b}$ to the simulated $D^{m}$-FSTD curves presented in Figures 3c and 3d, it can be observed that SINR accurately follows the comparative trend among different $m$ values. That said, although SINR provides a good match in general, slight discrepancies can be present when the BER values of different orders are very close (e.g., $\log _{10} M=10$ for $S_{r}=0.25$, between $m=2$ and $m=3$ ). This phenomenon is due to evaluating SINR with a significant underestimation of the true channel memory by selecting $L^{\prime}=10$. Overall, our results confirm that the comparative BER relationship between different derivative orders can be well-explained through SINR even with $L^{\prime}=10$, which is promising in terms of computational complexity in micro- to nano-scale machinery.

\subsection{Comparative Error Performance}

To show comparative error performance between FSTD and MaTD, Figures $3 \mathrm{c}$ and $3 \mathrm{~d}$ are presented respectively. Note that both detectors are memoryless and are of significantly low complexity. Considering their comparably low-complexity implementations to the schemes discussed in this paper, the conventional, energybased fixed threshold detector (FTD) [13] and the adaptive threshold detector (ATD) [3] are also presented in the figures.

The results of Figures $3 \mathrm{c}$ and $3 \mathrm{~d}$ show that FSTD outperforms MaTD in a large majority of scenarios. Furthermore, the results 


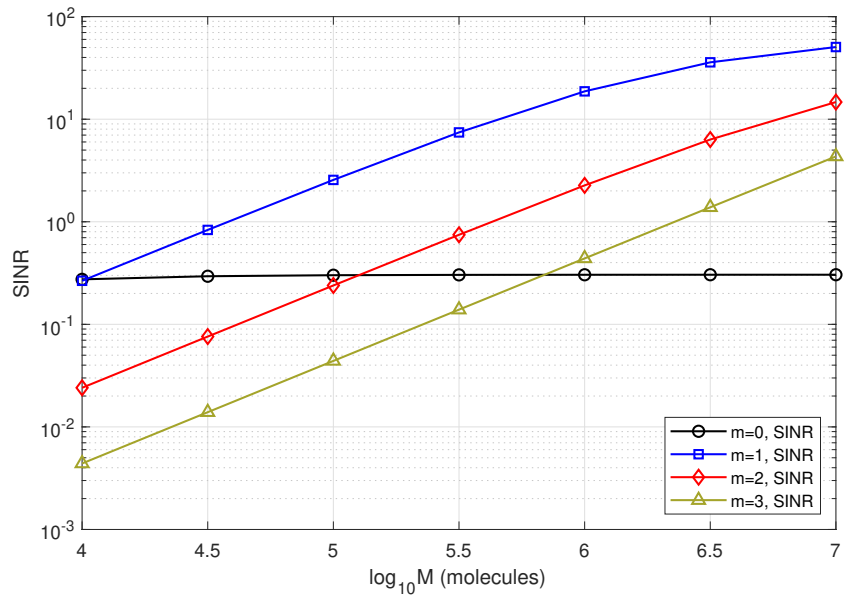

(a) SINR vs. $M, S_{r}=0.5, L^{\prime}=10$.

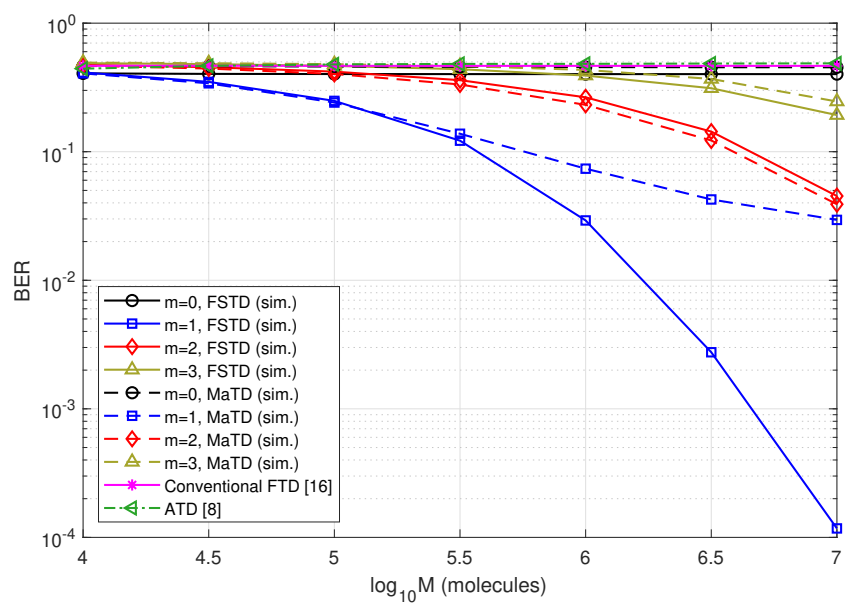

(c) Simulated BER vs. $M, S_{r}=0.5, L=100$.

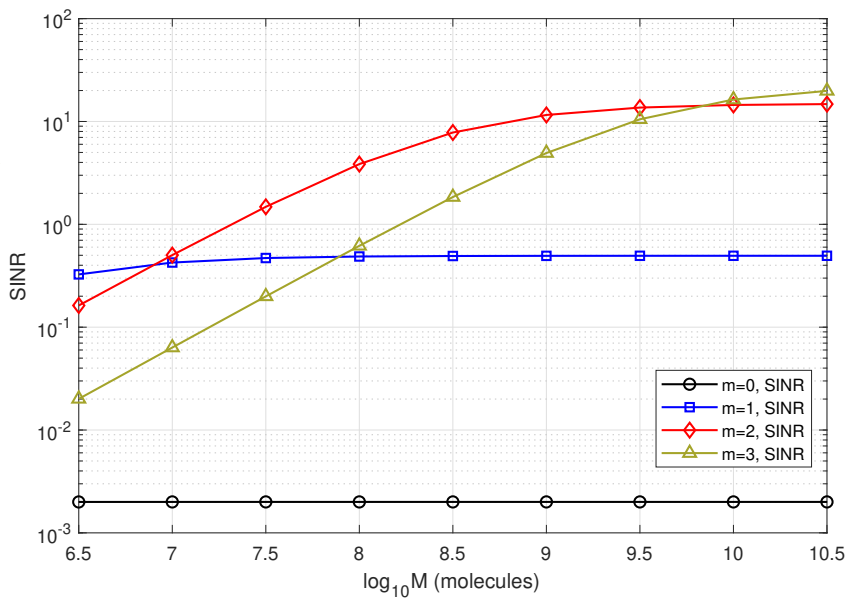

(b) SINR vs. $M, S_{r}=0.25, L^{\prime}=10$.

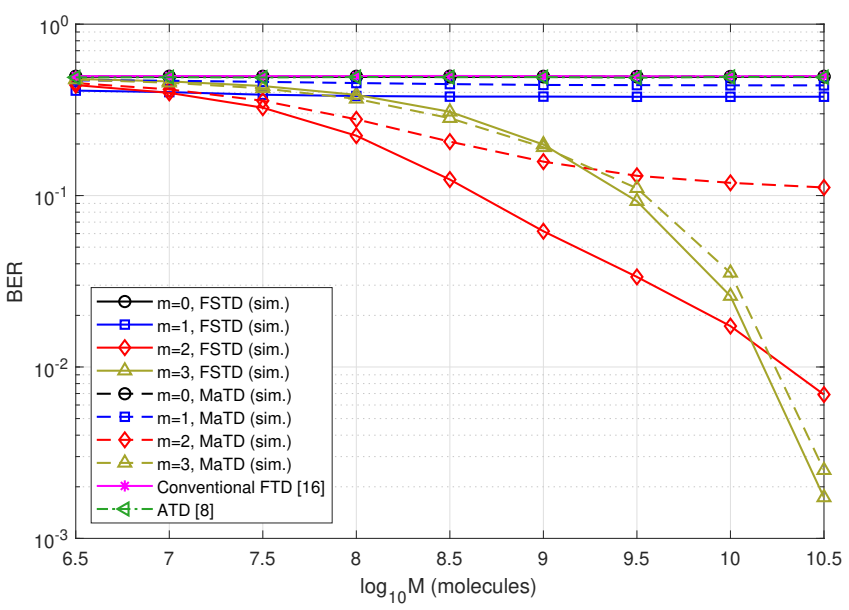

(d) Simulated BER vs. $M, S_{r}=0.25, L=200$.

Figure 3: BER and SINR vs. $M$. SNR $=10 \mathrm{~dB}, r_{0}=15 \mu \mathrm{m}, r_{r}=5 \mu \mathrm{m}, D=100 \mu \mathrm{m}^{2} \mathrm{~s}^{-1}, N=5 . \gamma$ values numerically optimized through exhaustive search.

show that derivative-based methods significantly outperform the non-derivative schemes (FTD, ATD, and $m=0$ schemes), which is due to the powerful ISI mitigation introduced by applying first or higher order derivative operations on the received signal. However, as also noted by [5], achieving this improvement requires that the transmitter is able to handle large transmission powers, in order to alleviate the effects of the noise amplification incurred by the $D^{m}$ operator.

\section{CONCLUSIONS}

In this paper, the fixed sample, fixed threshold detector (FSTD) has been generalized and proposed for use in conjunction with the higher order derivative-based pre-processor. For the $D^{m}$-FSTD pair, the derivative order $(m)$ optimization has been addressed through proposing an SINR-like cost function. Our numerical results show that the considered cost function accurately mirrors the comparative trend between different orders of $m$. Overall, with a proper selection of $m$, the $\boldsymbol{D}^{m}$-FSTD pair offers reliable communication under DBMC channels with extremely high ISI, providing a promising solution for achieving high data rate and low error rate DBMC.

\section{ACKNOWLEDGMENTS}

This work has been funded in part by one or more of the following grants: ONR N00014-15-1-2550, NSF CCF-1817200, ARO W911NF1910269, Cisco Foundation 1980393, DOE DE-SC0021417, Swedish Research Council 2018-04359, NSF CCF-2008927, ONR 503400-78050.

\section{REFERENCES}

[1] Gholamali Aminian, Hamidreza Arjmandi, Amin Gohari, Masoumeh NasiriKenari, and Urbashi Mitra. 2015. Capacity of diffusion-based molecular communication networks over LTI-Poisson channels. IEEE Transactions on Molecular, 
Biological, and Multi-Scale Communications 1, 2 (Nov. 2015), 188-201.

[2] Hamidreza Arjmandi, Amin Gohari, Masoumeh Nasiri Kenari, and Farshid Bateni. 2013. Diffusion-based nanonetworking: A new modulation technique and performance analysis. IEEE Communications Letters 17, 4 (Mar. 2013), 645-648.

[3] Martin Damrath and Peter Adam Hoeher. 2016. Low-complexity adaptive threshold detection for molecular communication. IEEE Transactions on NanoBioscience 15, 3 (2016), 200-208.

[4] Nariman Farsad, H. Birkan Yilmaz, Andrew Eckford, Chan-Byoung Chae, and Weisi Guo. 2016. A Comprehensive Survey of Recent Advancements in Molecular Communication. IEEE Communications Surveys \& Tutorials 18, 3 (Feb. 2016), 1887-1919.

[5] Mustafa Can Gursoy and Urbashi Mitra. 2020. Higher Order Derivatives: Improved Pre-Processing and Receivers for Molecular Communications. In Proc. IEEE Global Communications Conference (GLOBECOM). 1-6. https://doi.org/10. 1109/GLOBECOM42002.2020.9322240

[6] Mustafa Can Gursoy, Masoumeh Nasiri-Kenari, and Urbashi Mitra. 2021. Towards High Data-Rate Diffusive Molecular Communications: Performance Enhancement Strategies. Digital Signal Processing (Jul. 2021). https://doi.org/10.1016/j. dsp.2021.103161

[7] Mustafa Can Gursoy, Daewon Seo, and Urbashi Mitra. 2021. A ConcentrationTime Hybrid Modulation Scheme for Molecular Communications. IEEE Transactions on Molecular, Biological, and Multi-Scale Communications (2021), 1-1. https://doi.org/10.1109/TMBMC.2021.3071772

[8] Yu Huang, Xuan Chen, Miaowen Wen, Lie-Liang Yang, Chan-Byoung Chae, and Fei Ji. 2020. A Rising Edge-Based Detection Algorithm for MIMO Molecular Communication. IEEE Wireless Communications Letters 9, 4 (Apr. 2020), 523-527.

[9] Yu Huang, Fei Ji, Zhuangkun Wei, Miaowen Wen, Xuan Chen, Yuankun Tang, and Weisi Guo. 2021. Frequency Domain Analysis and Equalization for Molecular Communication. IEEE Transactions on Signal Processing 69 (Mar. 2021), 19521967.

[10] Vahid Jamali, Arman Ahmadzadeh, and Robert Schober. 2017. On the design of matched filters for molecule counting receivers. IEEE Communications Letters. 21, 8 (May 2017), 1711-1714.

[11] Deniz Kilinc and Ozgur B. Akan. 2013. Receiver Design for Molecular Communication. IEEE Journal on Selected Areas in Communications 31, 12 (Dec. 2013)
705-714.

[12] Na-Rae Kim and Chan-Byoung Chae. 2013. Novel modulation techniques using isomers as messenger molecules for nano communication networks via diffusion. IEEE Journal on Selected Areas in Communications 31, 12 (Jan. 2013), 847-856.

[13] Mehmet S Kuran, Huseyin Birkan Yilmaz, Tuna Tugcu, and Ian F Akyildiz. 2011. Modulation techniques for communication via diffusion in nanonetworks. In Proc. IEEE International Conference on Communications (ICC). 1-5.

[14] Reza Mosayebi, Amin Gohari, Mahtab Mirmohseni, and Masoumeh Nasiri-Kenari. 2018. Type-based sign modulation and its application for ISI mitigation in molecular communication. IEEE Transactions on Communications 66, 1 (Jan. 2018), 180-193.

[15] Adam Noel, Karen C. Cheung, and Robert Schober. 2014. Optimal Receiver Design for Diffusive Molecular Communication With Flow and Additive Noise. IEEE Transactions on NanoBioscience 13, 3 (Sept. 2014), 350-362.

[16] Adam Noel and Andrew W Eckford. 2017. Asynchronous peak detection for demodulation in molecular communication. In Proc. IEEE International Conference on Communications (ICC). IEEE, 1-6.

[17] Po-Jen Shih, Chia-Han Lee, Ping-Cheng Yeh, and Kwang-Cheng Chen. 2013. Channel Codes for Reliability Enhancement in Molecular Communication. IEEE Journal on Selected Areas in Communications 31, 12 (Dec. 2013), 857-867.

[18] Burcu Tepekule, Ali E. Pusane, Mehmet Şükrü Kuran, and Tuna Tugcu. 2015. A Novel Pre-Equalization Method for Molecular Communication via Diffusion in Nanonetworks. IEEE Communications Letters 19, 8 (Jun. 2015), 1311-1314.

[19] Tze-Yang Tung and Urbashi Mitra. 2019. Synchronization Error Robust Transceivers for Molecular Communication. IEEE Transactions Molecular, Biological, and Multi-Scale Communications 5, 3 (Dec. 2019), 207-221.

[20] Hao Yan, Ge Chang, Zhongke Ma, and Lin Lin. 2018. Derivative-Based Signal Detection for High Data Rate Molecular Communication System. IEEE Communications Letters. 22, 9 (Sep. 2018), 1782-1785.

[21] H Birkan Yilmaz and Chan-Byoung Chae. 2014. Arrival modelling for molecular communication via diffusion. IET Electronics Letters 50, 23 (Nov. 2014), 1667-1669.

[22] H Birkan Yilmaz, Akif Cem Heren, Tuna Tugcu, and Chan-Byoung Chae. 2014. Three-dimensional channel characteristics for molecular communications with an absorbing receiver. IEEE Communications Letters. 18, 6 (Jun. 2014), 929-932. 